University of Wollongong

Research Online

Faculty of Informatics - Papers (Archive)

Faculty of Engineering and Information

Sciences

June 2002

\title{
Modulated, perfect reconstruction filterbanks with integer coefficients
}

Alfred Mertins

University of Wollongong, mertins@uow.edu.au

T. Karp

Texas Tech University

Follow this and additional works at: https://ro.uow.edu.au/infopapers

Part of the Physical Sciences and Mathematics Commons

\section{Recommended Citation}

Mertins, Alfred and Karp, T.: Modulated, perfect reconstruction filterbanks with integer coefficients 2002. https://ro.uow.edu.au/infopapers/91

Research Online is the open access institutional repository for the University of Wollongong. For further information contact the UOW Library: research-pubs@uow.edu.au 


\title{
Modulated, perfect reconstruction filterbanks with integer coefficients
}

\begin{abstract}
We present design methods for perfect reconstruction (PR) integer-modulated filterbanks, including biorthogonal (low-delay) filterbanks. Both the prototype filter and the modulation sequences are composed of integers, thus allowing efficient hardware implementations and fast computation. To derive such filterbanks, we first start with the PR conditions known for cosine modulation and extend them to more general, integer modulation schemes. For the design of biorthogonal PR integer prototypes, a lifting strategy is introduced. To find suitable integer modulation schemes, new algebraic methods are presented. We show solutions where the PR conditions on the prototype filters and the modulation matrices are entirely decoupled and where some simple coupling is introduced. Both even and odd numbers of channels are considered. Design examples are presented for both cases.

\section{Disciplines}

Physical Sciences and Mathematics

\section{Publication Details}

This article was published as: Mertins, A \& Karp, T, Modulated, perfect reconstruction filterbanks with integer coefficients, IEEE Transactions on Signal Processing, June 2002, 50(6), 1398-1408. Copyright IEEE 2002
\end{abstract}




\title{
Modulated, Perfect Reconstruction Filterbanks With Integer Coefficients
}

\author{
Alfred Mertins, Member, IEEE, and Tanja Karp, Member, IEEE
}

\begin{abstract}
In this paper, we present design methods for perfect reconstruction (PR) integer-modulated filterbanks, including biorthogonal (low-delay) filterbanks. Both the prototype filter and the modulation sequences are composed of integers, thus allowing efficient hardware implementations and fast computation. To derive such filterbanks, we first start with the PR conditions known for cosine modulation and extend them to more general, integer modulation schemes. For the design of biorthogonal PR integer prototypes, a lifting strategy is introduced. To find suitable integer modulation schemes, new algebraic methods are presented. We show solutions where the PR conditions on the prototype filters and the modulation matrices are entirely decoupled and where some simple coupling is introduced. Both even and odd numbers of channels are considered. Design examples are presented for both cases.
\end{abstract}

Index Terms-Cosine modulation, filterbanks, interger coefficients, integer modulation, low delay, perfect reconstruction.

\section{INTRODUCTION}

$\mathbf{T}$ O IMPLEMENT a filterbank on a processor with finite-precision arithmetic, one usually needs to quantize the filter coefficients. This, however, results in loss of the perfect reconstruction (PR) property. It is therefore of significant interest to design filterbanks directly in such a way that PR can be achieved with integer arithmetic. Moreover, filterbanks operating with integer arithmetic allow for fast and efficient computation on signal processors and in dedicated hardware. For the case of two-channel filterbanks and associated wavelets, integer filters have been widely studied during the last decade. Integer linear-phase, biorthogonal, two-channel filterbanks and wavelets were found in [1] through the factorization of certain integer halfband filters. Paraunitary two-channel filterbanks with integer arithmetic were presented in [2]-[4]. More recently, a systematic design of two-channel filterbanks with integer arithmetic has been enabled by the lifting scheme presented in [5], [6]. For the case of $M$-channel modulated filterbanks, however, only very few design methods have been reported. The work in [7] presents solutions for the finite-precision implementation of the polyphase filters of paraunitary cosine-modulated filterbanks based on $\mu$-rotations. In [8], integer-coefficient prototypes for paraunitary cosine-modulated

\footnotetext{
Manuscript received February 7, 2001; revised February 12, 2002. The associate editor coordinating the review of this paper and approving it for publication was Dr. Masaaki Ikehara.

A. Mertins is with the School of Electrical, Computer, and Telecommunication Engineering, University of Wollongong, Wollongong, Australia (e-mail: mertins@uow.edu.au).

T. Karp is with the Department of Electrical and Computer Engineering, Texas Tech University, Lubbock, TX 79409-3102 USA (e-mail: tanja.karp@ttu.edu).

Publisher Item Identifier S 1053-587X(02)04390-8.
}

filterbanks were designed via a subspace approach. Design methods and implementation structures for PR filterbanks with integer modulation and integer prototypes have been presented in [9]-[12]. In [9], the integer modulation sequences are designed on the basis of the dyadic symmetry principle [13], and the filter lengths $L$ are restricted to the case $L=2 M$, where $M$ is the number of bands. In [10] and [11], prototypes with lengths $L \geq 2 M$ are considered for even $M$. The work in [12] considers the sum-of-powers-of-two implementation of the prototype and the cosine modulation. Further design methods for integer DCT matrices suitable for use in integer filterbanks have been presented in [14]. Thus far, all solutions reported in the literature consider an even number of channels.

In this paper, we generalize the theory of integer-modulated filterbanks to an arbitrary number of channels (odd and even) and a delay of the form $D=2 s M+2 M-1$ with an arbitrary integer $s$. For the design of biorthogonal PR integer prototypes, a lifting strategy is introduced. To find suitable integer modulation schemes, new algebraic methods are presented. We first apply the method in [13] to the design of modulation matrices, and then, we present a projection-based method. We derive solutions where the PR conditions on the prototype and the modulation are entirely decoupled and where some simple coupling is introduced. In the coupled case, the requirements on the prototype and the modulation sequences can be traded off between the two, resulting in an increased design freedom compared with the decoupled case. Design examples will be presented for both even and odd numbers of channels.

Notation: $I_{M}$ and $J_{M}$ denote the $M \times M$ identity and counter identity matrices, respectively. The expression \lceil\rceil denotes the ceiling operation. Similarly, \lfloor\rfloor means the floor operations. The term $\delta_{i, j}$ denotes the Kronecker symbol. Matrix entries are counted starting from zero, i.e., $[C]_{0,0}$ is the element in the upper left corner of matrix $C$.

\section{COSINE-MODUlated FilterbanKS}

\section{A. Review of Perfect Reconstruction Conditions}

In cosine-modulated filterbanks, the analysis and synthesis filters $h_{k}(n)$ and $g_{k}(n), k=0, \ldots, M-1$ are derived from lowpass prototypes $p(n)$ and $q(n)$ as

$$
h_{k}(n)=p(n) c_{1, k}(n), \quad g_{k}(n)=q(n) c_{2, k}(n)
$$

where $c_{1, k}(n)$ and $c_{2, k}(n)$ are cosine sequences providing the modulation. The range for $n$ in (1) depends on the filter lengths that may be different for the analysis and synthesis sides and are, in general, independent of the delay of the entire analysis/synthesis system. Fig. 1 shows a straightforward structure for the 


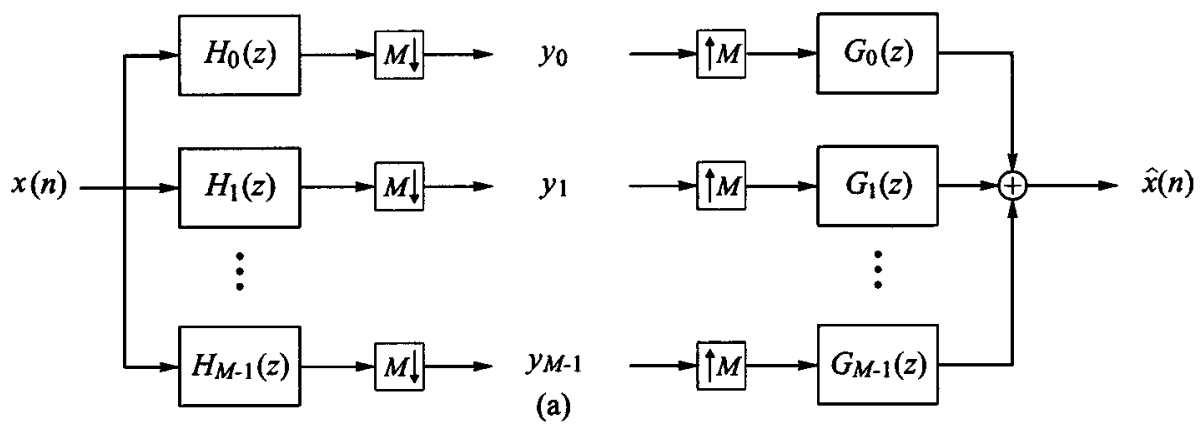

(a)

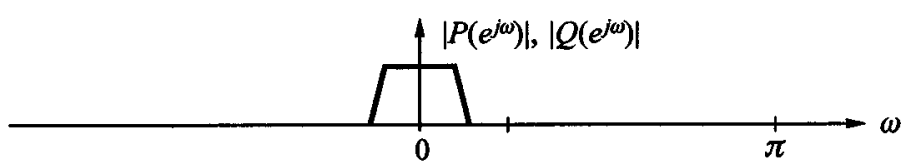

(b)

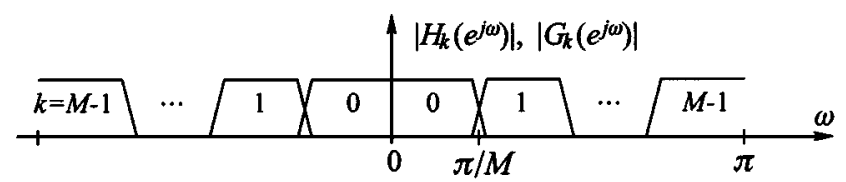

(c)

Fig. 1. Cosine-modulated filterbank. (a) Structure. (b) Prototype frequency responses. (c) Frequency responses of analysis and synthesis filters.

filterbank and illustrates the frequency responses of the filters. Various modulation schemes and filter design strategies have been proposed in the literature, including FIR and IIR solutions, critical sampling and oversampling, even and odd numbers of channels, paraunitary, and biorthogonal, low-delay filterbanks [15]-[21]. A reason for the popularity of cosine-modulated filterbanks is the fact that they can be implemented very efficiently in polyphase structure [15].

In this paper, we consider critical subsampling and a general number of channels $(M)$ that may be even or odd, and the following definition of cosine sequences [21]:

$$
\begin{gathered}
c_{1, k}(n)=2 \cos \left[\frac{\pi}{M}\left(k+\frac{1}{2}\right)\left(n-\frac{D}{2}\right)+(-1)^{k} \pi / 4\right] \\
c_{2, k}(n)=2 \cos \left[\frac{\pi}{M}\left(k+\frac{1}{2}\right)\left(n-\frac{D}{2}\right)-(-1)^{k} \pi / 4\right] \\
k=0 \ldots, M-1 .
\end{gathered}
$$

The overall delay $(D)$ of the analysis/synthesis system is assumed to be of the form

$$
D=2 s M+2 M-1
$$

where $s$ is an integer.

To explain the PR conditions, the polyphase matrices $\boldsymbol{E}(z)$ and $\boldsymbol{R}(z)$ of the analysis and synthesis filterbanks are introduced as [21]

$$
\begin{aligned}
& \boldsymbol{E}(z)=\boldsymbol{C}_{1}\left[\begin{array}{c}
\boldsymbol{P}_{0}\left(z^{2}\right) \\
z^{-1} \boldsymbol{P}_{1}\left(z^{2}\right)
\end{array}\right] \\
& \boldsymbol{R}(z)=\left[z^{-1} \boldsymbol{Q}_{1}\left(z^{2}\right), \quad \boldsymbol{Q}_{0}\left(z^{2}\right)\right] \boldsymbol{C}_{2}^{T}
\end{aligned}
$$

with

$$
\left[\boldsymbol{C}_{1}\right]_{k, n}=c_{1, k}(n), \quad\left[C_{2}\right]_{k, 2 M-1-n}=c_{2, k}(n)
$$

for $k=0,1, \ldots, M-1, n=0,1, \ldots, 2 M-1$, and

$$
\begin{aligned}
& P_{0}\left(z^{2}\right)=\operatorname{diag}\left[P_{0}\left(-z^{2}\right), \ldots, P_{M-1}\left(-z^{2}\right)\right] \\
& \boldsymbol{Q}_{0}\left(z^{2}\right)=\operatorname{diag}\left[Q_{M-1}\left(-z^{2}\right), \ldots, Q_{0}\left(-z^{2}\right)\right] \\
& P_{1}\left(z^{2}\right)=\operatorname{diag}\left[P_{M}\left(-z^{2}\right), \ldots, P_{2 M-1}\left(-z^{2}\right)\right] \\
& \boldsymbol{Q}_{1}\left(z^{2}\right)=\operatorname{diag}\left[Q_{2 M-1}\left(-z^{2}\right), \ldots, Q_{M}\left(-z^{2}\right)\right] .
\end{aligned}
$$

The terms $P_{j}(z)$ and $Q_{j}(z)$ in (6) are the type-1 polyphase components of the prototypes

$$
\begin{aligned}
P_{j}(z) & =\sum_{\ell} p(2 \ell M+j) z^{-\ell} \\
Q_{j}(z) & =\sum_{\ell} q(2 \ell M+j) z^{-\ell}, \quad j=0, \ldots, 2 M-1 .
\end{aligned}
$$

With $\boldsymbol{E}(z)$ and $\boldsymbol{R}(z)$, the conditions for perfect reconstruction with a delay $D=2 s M+2 M-1$ can now be formulated as [15]

$$
\boldsymbol{R}(z) \boldsymbol{E}(z)=z^{-2 s-1} \boldsymbol{I}_{M} .
$$

Using the fact that

$$
C_{2}^{\mathrm{T}} \boldsymbol{C}_{1}=2 M\left[\begin{array}{cc}
(-1)^{s} \boldsymbol{I}_{M}+\boldsymbol{J}_{M} & \mathbf{0} \\
\mathbf{0} & (-1)^{s} \boldsymbol{I}_{M}-\boldsymbol{J}_{M}
\end{array}\right]
$$

and by substitution of $z$ for $-z^{2}$, the PR constraints (8) can be rewritten as [21]

$$
\begin{aligned}
Q_{k}(z) & P_{2 M-1-k}(z)+Q_{M+k}(z) P_{M-1-k}(z)=\frac{1}{2 M} z^{-s} \\
k & =0, \ldots, M-1 \\
Q_{k}(z) & =\lambda_{k} z^{-a_{k}} P_{k}(z), \quad Q_{M+k}(z)=\lambda_{k} z^{-a_{k}} P_{M+k}(z) \\
k & =0, \ldots, M-1 .
\end{aligned}
$$


To obtain contiguous prototypes, we assume the delays $a_{k}$ to be zero. By combining (10) and (11), we then obtain the conditions

$$
\lambda_{k}\left[P_{k}(z) P_{2 M-1-k}(z)+P_{M+k}(z) P_{M-1-k}(z)\right]=\frac{1}{2 M} z^{-s}
$$

on the analysis prototype, which need to be satisfied for $k=$ $0, \ldots,\lceil M / 2\rceil-1.1^{1}$ Given an analysis prototype that satisfies (12), the synthesis prototype is to be constructed according to (11).

Note that (12) shows the PR conditions on the prototype for critically sampled, biorthogonal, cosine-modulated filterbanks with the delay in (3). In the special case of paraunitary filterbanks, a linear phase (symmetric) prototype is required, and it needs to be used for both analysis and synthesis. Equations (10) and (11) then lead to the following conditions on paraunitary prototypes:

$$
\begin{gathered}
P_{k}(z) P_{k}\left(z^{-1}\right)+P_{M+k}(z) P_{M+k}\left(z^{-1}\right)=\frac{1}{2 M}, \\
k=0, \ldots,\left\lceil\frac{M}{2}\right\rceil-1 .
\end{gathered}
$$

\section{B. Periodicities and Symmetries of Cosine Sequences}

In this section, we analyze the periodicities and symmetries found in the cosine sequences $c_{\ell, k}(n), \ell=1,2$ and describe how these properties can be utilized to construct and implement the cosine matrices $\boldsymbol{C}_{\ell}, \ell=1,2$ in an efficient manner. In Section III, this description will then be used to derive more general modulation schemes.

Using the properties $\cos (\varphi)=\cos (\varphi+\kappa 2 \pi), \kappa \in \mathbb{Z}$, and $\cos (\varphi)=-\cos (\varphi+\pi)$, it follows from (2) that the cosine sequences have the following periodicities:

$$
\begin{aligned}
c_{\ell, k}(n+\kappa 4 M) & =c_{\ell, k}(n) \\
c_{\ell, k}(n+\kappa 4 M+2 M) & =-c_{\ell, k}(n)
\end{aligned}
$$

for $\ell=1,2$ and $\kappa \in \mathbb{Z}$. Obviously, (14) means that the cosine sequences have period $4 M$, and (15) shows that the sequences have half periods that are equal up to the sign. Both properties (14) and (15) have already been taken into account when formulating the PR conditions in Section II-A.

To describe symmetries within the sequences, we first define the value

$$
\mu=\left\lfloor\frac{M}{2}\right\rfloor .
$$

For even $M$, one can infer from (2) that

$$
c_{\ell, k}(n+\mu)=(-1)^{s} c_{\ell, k}(\mu-n-1)
$$

and for odd $M$, one obtains

$$
c_{\ell, k}(n+\mu)=(-1)^{s} c_{\ell, k}(\mu-n) .
$$

${ }^{1}$ Note that the conditions for $k=\lceil M / 2\rceil, \ldots, M-1$ are included in the ones for $k=0, \ldots,\lceil M / 2\rceil-1$.
By taking the properties (14), (15), (17), and (18) into account, one can see that the cosine sequences can be derived through symmetric, antisymmetric, and periodic extensions of the values $c_{\ell, k}(n)$ for $n=\mu, \mu+1, \ldots, \mu+M-1$. The matrices $C_{1}$ and $\boldsymbol{C}_{2}$ can thus be described as

$$
C_{1}=V^{c} Y_{1}, \quad C_{2}=V^{c} Y_{2}
$$

with

$$
\left[\boldsymbol{V}^{c}\right]_{k, n}=c_{1, k}(n+\mu), \quad k, n=0,1, \ldots, M-1
$$

where $Y_{\ell}$ are sparse $M \times 2 M$ matrices with entries \pm 1 and 0 , whose purpose is to symmetrically extend the columns of the $M \times M$ matrix $\boldsymbol{V}^{c}$.

Equation (19) can also be seen as a key to a fast implementation of the modulation within a polyphase realization of the filterbank. Polyphase realizations have, e.g., been discussed in [15] and [22]. For example, for the realization of the analysis filterbank in polyphase form, the factorization (19) requires only the multiplication of the polyphase filter outputs with the sparse matrix $\boldsymbol{Y}_{1}$ (requiring only additions and subtractions) and a multiplication with the $M \times M$ cosine matrix $V^{c}$ instead of a multiplication with the $M \times 2 M$ matrix $C_{1}$. The same efficiency can be obtained on the synthesis side.

To describe the matrices $\boldsymbol{Y}_{\ell}, \ell=1,2$ and the properties of $V^{c}$ in detail, we will have to distinguish between even and odd $M$ and $s$.

Even $M$ and arbitrary $s$. For even $M$, one finds that

$$
\begin{aligned}
& \boldsymbol{Y}_{1}=\left[\begin{array}{cccc}
(-1)^{s} \boldsymbol{J}_{M / 2} & \boldsymbol{I}_{M / 2} & \mathbf{0} & \mathbf{0} \\
\mathbf{0} & \mathbf{0} & \boldsymbol{I}_{M / 2} & -(-1)^{s} \boldsymbol{J}_{M / 2}
\end{array}\right] \\
& \boldsymbol{Y}_{2}=(-1)^{s} \boldsymbol{Y}_{1}
\end{aligned}
$$

and $\boldsymbol{V}^{c}$ satisfies $\left[\boldsymbol{V}^{c}\right]^{T} \boldsymbol{V}^{c}=2 M \boldsymbol{I}$.

Odd $M$ and even $s$. To describe $\boldsymbol{Y}_{1}$ and $\boldsymbol{Y}_{2}$ for odd $M$, it is convenient to express the matrices element wise. They are

$$
\begin{aligned}
{\left[Y_{1}\right]_{i, k} } & =\left[Y_{2}\right]_{i, k} \\
& = \begin{cases}\delta_{i, \mu-k}, & k=0, \ldots, \mu \\
\delta_{i, k-\mu}, & k=\mu+1, \ldots, M+\mu-1 \\
0, & k=M+\mu \\
-\delta_{i, 2 M+\mu-k}, & k=M+\mu+1, \ldots, 2 M-1\end{cases}
\end{aligned}
$$

with $i=0, \ldots, M-1$. The matrix $V^{c}$ satisfies $\left[V^{c}\right]^{T} V^{c}=$ $2 M \operatorname{diag}[2,1, \ldots, 1]$.

Odd $M$ and odd $s$. We have

$$
\begin{aligned}
{\left[\boldsymbol{Y}_{1}\right]_{i, k} } & = \begin{cases}-\delta_{i, \mu-k-1}, & k=0, \ldots, \mu-1 \\
0, & k=\mu \\
\delta_{i, k-\mu-1}, & k=\mu+1, \ldots, M+\mu \\
\delta_{i, 2 M+\mu-k-1}, & k=M+\mu+1, \ldots, 2 M-1\end{cases} \\
\boldsymbol{Y}_{2} & =-\boldsymbol{Y}_{1}
\end{aligned}
$$

for $i=0, \ldots, M-1$ and $\left[V^{c}\right]^{T} V^{c}=2 M \operatorname{diag}[1, \ldots, 1,2]$. 
Remark: An alternative way to describe $\boldsymbol{C}_{1}$ and $\boldsymbol{C}_{2}$ is given by

$$
C_{1}=U^{c} \boldsymbol{X}_{1}, \quad C_{2}=\boldsymbol{U}^{c} \boldsymbol{X}_{2}
$$

with

$$
\begin{aligned}
& \boldsymbol{X}_{1}=\left[\boldsymbol{I}_{M}+(-1)^{s} \boldsymbol{J}_{M}, \boldsymbol{I}_{M}-(-1)^{s} \boldsymbol{J}_{M}\right] \\
& \boldsymbol{X}_{2}=(-1)^{s} \boldsymbol{X}_{1}
\end{aligned}
$$

where $\boldsymbol{U}^{c}$ is the $M \times M$ DCT-IV matrix [15]. This way of representing $\boldsymbol{C}_{1}$ and $\boldsymbol{C}_{2}$ has been used in [23] to relax the conditions on the modulation and in [10] to design certain integer-modulated filterbanks. However, for the implementation of $\boldsymbol{C}_{1}$ and $\boldsymbol{C}_{2}$, it is advantageous to use (19) instead of (24) because the multiplication with $\boldsymbol{Y}_{\ell}$ requires fewer operations than the multiplication with $\boldsymbol{X}_{\ell}, \ell=1,2$.

\section{PR Conditions For General Modulation SeQuences}

In this section, we discuss possibilities of replacing the $M \times$ $2 M$ cosine-modulation matrices $\boldsymbol{C}_{1}$ and $\boldsymbol{C}_{2}$ in (4) with more general transform matrices $\boldsymbol{T}_{1}$ and $\boldsymbol{T}_{2}$ while maintaining PR. In other words, the cosine sequences $c_{1, k}(n)$ and $c_{2, k}(n)$ get replaced with more general sequences $t_{1, k}(n)$ and $t_{2, k}(n)$, where $\left[\boldsymbol{T}_{1}\right]_{k, n}=t_{1, k}(n)$, and $\left[\boldsymbol{T}_{2}\right]_{k, 2 M-1-n}=t_{2, k}(n)$. We will first look at solutions where the PR conditions on the prototype and the modulation are completely decoupled, as it is the case with the original cosine modulation. The product $\boldsymbol{T}_{2}^{T} \boldsymbol{T}_{1}$ then has to be equal to the right-hand side of (9) up to a scale factor. A more general formulation that couples the requirements on the modulation matrices and the prototype filters in a simple way will be described in Section III-B. This approach has the advantage of increasing the design freedom.

The proposed design methods for $\boldsymbol{T}_{1}$ and $\boldsymbol{T}_{2}$ are based on the factorizations

$$
T_{1}=V Y_{1}, \quad T_{2}=V Y_{2}
$$

which correspond to the factorizations of the cosine matrices in (19). The matrix $V$ contains the free parameters to be optimized in order to meet certain design criteria such as a desired frequency response of the modulated filters and/or integer-valued transform matrices. The condition on $V$ can be formulated as

$$
\boldsymbol{V}^{T} \boldsymbol{V}=\boldsymbol{\Gamma}
$$

where $\boldsymbol{\Gamma}$ is a diagonal matrix that will be further specified in the following. ${ }^{2}$ Due to sparseness and the special entries of $\boldsymbol{Y}_{\ell}$, the matrices $\boldsymbol{T}_{\ell}, \ell=1,2$ can also be implemented more efficiently in factored than in direct form.

\section{A. Decoupled Conditions}

The PR conditions on the prototype and the modulation sequences remain decoupled, as for cosine modulation, if $\boldsymbol{T}_{1}$ and $T_{2}$ satisfy

$$
\boldsymbol{T}_{2}^{\mathrm{T}} \boldsymbol{T}_{1}=\varepsilon\left[\begin{array}{cc}
(-1)^{s} \boldsymbol{I}_{M}+\boldsymbol{J}_{M} & \mathbf{0} \\
\mathbf{0} & (-1)^{s} \boldsymbol{I}_{M}-\boldsymbol{J}_{M}
\end{array}\right] .
$$

${ }^{2}$ To obtain a more general approach, one may use different matrices for the analysis and synthesis sides and define $T_{\ell}=V_{\ell} Y_{\ell}, \ell=1,2$ with $V_{2}^{T} V_{1}=$ $\boldsymbol{\Gamma}$. However, we will consider $\boldsymbol{V}_{2}=\boldsymbol{V}_{1}$ throughout this paper.
By relaxing the condition (8) to perfect reconstruction up to a scale factor $\gamma$

$$
\boldsymbol{R}(z) \boldsymbol{E}(z)=z^{-2 s-1} \gamma \boldsymbol{I}_{M},
$$

the conditions (12) on the prototype become

$$
\begin{gathered}
\lambda_{k}\left[P_{k}(z) P_{2 M-1-k}(z)+P_{M+k}(z) P_{M-1-k}(z)\right]=\frac{\gamma}{\varepsilon} z^{-s} \\
k=0, \ldots,\left\lceil\frac{M}{2}\right\rceil-1 .
\end{gathered}
$$

The comparison with the properties of the cosine matrices shows that $\boldsymbol{V}$ then needs to satisfy (27) with $\boldsymbol{\Gamma}$, as specified in the following.

Even $M$ and arbitrary $s$

$$
\boldsymbol{\Gamma}=\varepsilon \boldsymbol{I}_{M}
$$

Odd $M$ and even $s$

$$
\boldsymbol{\Gamma}=\varepsilon \cdot \operatorname{diag}[2,1, \ldots, 1] .
$$

Odd $M$ and odd $s$

$$
\boldsymbol{\Gamma}=\varepsilon \cdot \operatorname{diag}[1, \ldots, 1,2] .
$$

Note that the use of integer modulation matrices and integer filter coefficients makes the introduction of scale factors $\varepsilon$ and $\gamma$ necessary. From (27) and (31) with $\boldsymbol{V}$ being an integer matrix, it is clear that $\varepsilon$ is an integer. To have PR with integer filters, $\gamma$ and $\gamma / \varepsilon$ must also be integers. The values $\lambda_{k}$ do not necessarily have to be integers, as long as $P(z)$ and $Q(z)$ have integer coefficients.

Remark: The parameterization of $\boldsymbol{T}_{1}$ and $\boldsymbol{T}_{2}$ in the form

$$
\boldsymbol{T}_{1}=\boldsymbol{U} \boldsymbol{X}_{1}, \quad \boldsymbol{T}_{2}=\boldsymbol{U} \boldsymbol{X}_{2}
$$

similar to (24) yields the requirement

$$
\boldsymbol{U}^{T} \boldsymbol{U}=\frac{\varepsilon}{2} \boldsymbol{I}_{M}
$$

where $\varepsilon / 2$ is an integer. It is easy to show that all matrices $T_{\ell}$, $\ell=1,2$ designed according to (34) can alternatively be implemented with the factorization (26). For this, given $\boldsymbol{T}_{1}$ and $\boldsymbol{T}_{2}$, one has to construct $\boldsymbol{V}$, as $\boldsymbol{V}=\boldsymbol{T}_{\ell} \boldsymbol{Y}_{\ell}^{+}$, where $\boldsymbol{Y}_{\ell}^{+}$is the pseudo inverse of $Y_{\ell}$. Explicit expressions for $Y_{\ell}^{+}$are listed in the following. Because of (35), however, integer matrices $\boldsymbol{T}_{\ell}$ designed according to (26) can only be implemented in the form (34) if $\varepsilon / 2$ is an integer.

\section{Even $M$ and arbitrary $s$ :}

$$
\boldsymbol{Y}_{1}^{+}=\frac{1}{2} \boldsymbol{Y}_{1}^{T}, \quad \boldsymbol{Y}_{2}^{+}=\frac{1}{2} \boldsymbol{Y}_{2}^{T} .
$$

Odd $M$, even $s, i=0, \ldots, 2 M-1, k=0, \ldots, M-1$ :

$$
\left[\boldsymbol{Y}_{1}^{+}\right]_{i, k}=\left[\boldsymbol{Y}_{2}^{+}\right]_{i, k}= \begin{cases}1, & i=\mu, k=0 \\ \frac{1}{2}\left[Y_{1}\right]_{k, i}, & \text { otherwise. }\end{cases}
$$

Odd $M$, odd $s, i=0, \ldots, 2 M-1, k=0, \ldots, M-1$ :

$$
\begin{aligned}
{\left[\boldsymbol{Y}_{1}^{+}\right]_{i, k} } & =-\left[\boldsymbol{Y}_{2}^{+}\right]_{i, k} \\
& = \begin{cases}1, & i=M+\mu, k=M-1 \\
\frac{1}{2}\left[\boldsymbol{Y}_{1}\right]_{k, i}, & \text { otherwise. }\end{cases}
\end{aligned}
$$


Remark: Due to the parameterization (26) for $\boldsymbol{T}_{1}$ and $\boldsymbol{T}_{2}$, the modulation sequences $t_{1, k}(n)$ and $t_{2, k}(n)$ are derived though a periodic extension of length- $M$ sequences stored in the rows of $\boldsymbol{V}$. Thus, (26) describes a complete parameterization for all general (including integer) modulation sequences that obey the same symmetry rules as the cosine sequences, which are expressed in (17) and (18). Alternatively, one may design $\boldsymbol{T}_{1}$ and $\boldsymbol{T}_{2}$ directly to satisfy (28). In this case, half periods of the sequences $t_{\ell, k}(n)$ would be designed directly without additionally imposing symmetries and antisymmetries. This may increase the design freedom for integer solutions, but it also increases the implementation cost because sparse factorization, as in (26) or (34), is no longer possible. For this reason, we will not pursue such a design in the following.

\section{B. Coupled Conditions}

We consider the formulation for $T_{1}$ and $T_{2}$ in (26) and relax the conditions on $\boldsymbol{V}$ in (27) to a more general matrix $\boldsymbol{\Gamma}$. Again, to further specify $\boldsymbol{\Gamma}$, we need to distinguish between even and odd $M$ and $s$.

Even $M$ and arbitrary $s$ : For even $M$, we define $\boldsymbol{\Gamma}$ as

$$
\boldsymbol{\Gamma}=\operatorname{diag}\left[w_{(M / 2)-1}, \ldots, w_{0}, w_{0}, \ldots, w_{(M / 2)-1}\right]
$$

where the values $w_{k}$ are arbitrary, nonzero, real-valued numbers that need to be finally specified in accordance with the prototypes' properties. With $\boldsymbol{\Gamma}$ as in (36), the product $\boldsymbol{T}_{2}^{T} \boldsymbol{T}_{1}$ takes on the form

$$
T_{2}^{T} \boldsymbol{T}_{1}=\left[\begin{array}{cc}
\Delta\left[(-1)^{s} \boldsymbol{I}_{M}+\boldsymbol{J}_{M}\right] & \mathbf{0} \\
\mathbf{0} & \boldsymbol{\Delta}\left[(-1)^{s} \boldsymbol{I}_{M}-\boldsymbol{J}_{M}\right]
\end{array}\right]
$$

with

$$
\boldsymbol{\Delta}=\operatorname{diag}\left[w_{0}, \ldots, w_{(M / 2)-1}, w_{(M / 2)-1}, \ldots, w_{0}\right] .
$$

Following the same derivation as in Section II, we obtain the following PR conditions on the prototype:

$$
\begin{gathered}
w_{k} \lambda_{k}\left[P_{k}(z) P_{2 M-1-k}(z)+P_{M+k}(z) P_{M-1-k}(z)\right]=\gamma z^{-s} \\
k=0, \ldots,\left\lceil\frac{M}{2}\right\rceil-1
\end{gathered}
$$

The factors $w_{k}$ need to be found during a joint design of prototypes and modulation matrices such that both (36) and (39) are satisfied.

Odd $M$ and even $s$ : For this case we choose

$$
\begin{gathered}
\boldsymbol{\Gamma}=\operatorname{diag}\left[2 w_{\lfloor M / 2\rfloor}, w_{\lfloor M / 2\rfloor-1}, \ldots\right. \\
\left.w_{0}, w_{0}, \ldots, w_{\lfloor M / 2\rfloor-1}\right] .
\end{gathered}
$$

Again, the product $\boldsymbol{T}_{2}^{T} \boldsymbol{T}_{1}$ takes on the form (37) but, now, with

$$
\boldsymbol{\Delta}=\operatorname{diag}\left[w_{0}, \ldots, w_{\lfloor M / 2\rfloor-1}, \begin{array}{l}
w_{\lfloor M / 2\rfloor} \\
\left.w_{\lfloor M / 2\rfloor-1}, \ldots, w_{0}\right] .
\end{array}\right.
$$

The PR conditions on the prototype remain the same as in (39).

Odd $M$ and odd $s$ : We choose

$$
\begin{array}{r}
\boldsymbol{\Gamma}=\operatorname{diag}\left[w_{\lfloor M / 2\rfloor-1}, \ldots, w_{0}, w_{0}, \ldots,\right. \\
\left.w_{\lfloor M / 2\rfloor-1}, 2 w_{\lfloor M / 2\rfloor}\right] .
\end{array}
$$

The product $\boldsymbol{T}_{2}^{T} \boldsymbol{T}_{1}$ takes on the form (37) with $\boldsymbol{\Delta}$ as in (41). The PR conditions on the prototype remain the same as in (39).

The introduction of the factors $w_{k}$ gives us increased design freedom compared with the decoupled conditions in Section III-A because it allows scale factors to be traded off between the prototype and the modulation.

\section{Design of InTEger-CoefFicient Prototype FiLTERS USING A LIFTING SCHEME}

In this section, we discuss a new method for the design of integer-coefficient prototypes in general, including low-delay integer-modulated filterbanks. The method principally yields prototypes for biorthogonal filterbanks. For the design of linearphase integer prototypes, see [8]. The conditions (30) for the decoupled and (39) for the coupled design can be interpreted as PR conditions on two-channel filterbanks without subsampling; see Fig. 2(a). It is well known that such structures can be realized using lifting schemes [5]. Fig. 2(b) shows the equivalent lifting structure. Each lifting step in the analysis filterbank in Fig. 2(b) consists of a possible delay by $\rho_{k, l}$ samples and a multiplication with a scalar $a_{k, l}$ in the upper branch; then, a filtered version of the lower branch is added to the upper branch, and the lower branch is also weighted by the factor $a_{k, l}$. Finally, the lower and upper branches are crossed, which allows for the use of a single type of lifting, instead of requiring lifting and dual lifting steps as in [5]. The corresponding lifting step in the synthesis filterbank implements the inverse operation, except for the scaling. The polyphase filters then are expressed as

$$
\begin{aligned}
& {\left[\begin{array}{c}
P_{k}(z) \\
P_{M+k}(z)
\end{array}\right]} \\
& \quad=\prod_{l=1}^{\nu}\left(\left[\begin{array}{ll}
0 & 1 \\
1 & 0
\end{array}\right]\left[\begin{array}{cc}
a_{k, l} z^{-\rho_{k, l}} & B_{k, l}(z) \\
0 & a_{k, l}
\end{array}\right]\right)\left[\begin{array}{l}
1 \\
1
\end{array}\right] \\
& {\left[\begin{array}{ll}
P_{2 M-1-k}(z) & P_{M-1-k}(z)
\end{array}\right]} \\
& \quad=\left[\begin{array}{ll}
1 & 1
\end{array}\right] \prod_{l=\nu}^{1}\left(\left[\begin{array}{cc}
a_{k, l} & -B_{k, l}(z) \\
0 & a_{k, l} z^{-\rho_{k, l}}
\end{array}\right]\left[\begin{array}{ll}
0 & 1 \\
1 & 0
\end{array}\right]\right) .
\end{aligned}
$$

It can be easily verified that

$$
\begin{array}{r}
{\left[\begin{array}{ll}
P_{2 M-1-k}(z) & P_{M-1-k}(z)
\end{array}\right]\left[\begin{array}{c}
P_{k}(z) \\
P_{M+k}(z)
\end{array}\right]} \\
=2 \prod_{l=1}^{\nu} a_{k, l}^{2} z^{-\rho_{k, l}}
\end{array}
$$

To satisfy the PR constraints (30) or (39), the values for $\rho_{k, l}$ and $a_{k, l}, k=0, \ldots,\lceil M / 2\rceil-1, l=1, \ldots, \nu$ have to fulfill

$$
\begin{aligned}
\sum_{l=1}^{\nu} \rho_{k, l} & =s \\
2 \prod_{l=1}^{\nu} a_{k, l}^{2} & = \begin{cases}\frac{\gamma}{\varepsilon \lambda_{k}}, & \text { for the uncoupled design } \\
\frac{\gamma}{w_{k} \lambda_{k}}, & \text { for the coupled design. }\end{cases}
\end{aligned}
$$

The system functions $B_{k, l}(z)$ can be freely chosen. They influence the frequency response and the length of the prototype 


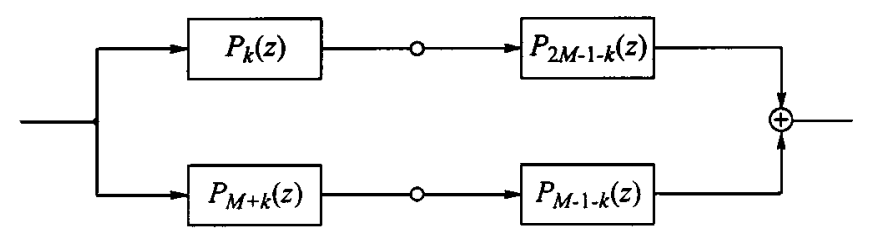

(a)

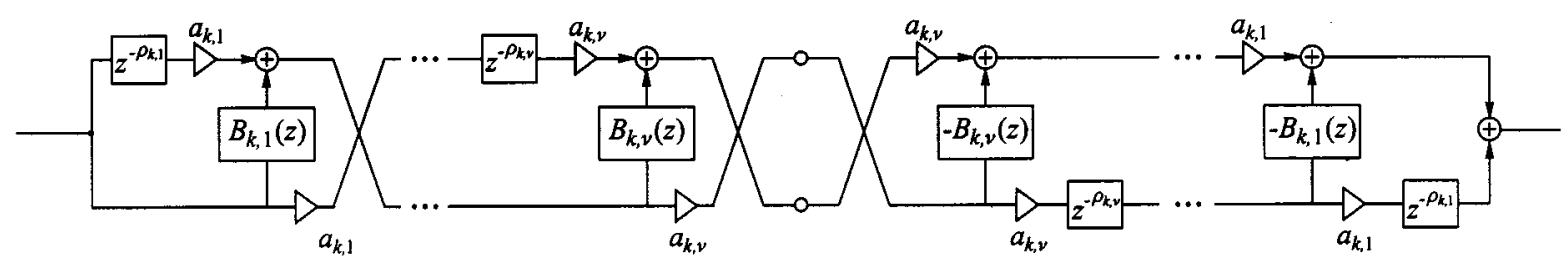

(b)

Fig. 2. Equivalent structures for expressing the PR conditions. (a) Direct structure. (b) Lifting structure. Both structures need to have the overall system function $\left(\gamma / \varepsilon \lambda_{k}\right) z^{-s}$ for the decoupled and $\left(\gamma / w_{k}, \lambda_{k}\right) z^{-s}$ for the coupled case.

filter but not the PR behavior. In addition, $a_{k, l}$ and $\rho_{k, l}$ are free design parameters, but they need to satisfy (46).

Quantization of the coefficients of $B_{k, l}(z)$ does not alter the PR property as long as it is applied in the same way in the analysis and synthesis filterbank because apart from sign changes, the same coefficients occur on the analysis and synthesis sides. Using only integer values for the coefficients of $B_{k, l}(z)$ and the factors $a_{k, l}$ automatically yields integer coefficient prototype filters.

To obtain a set of integer coefficients, we apply the following algorithm.

Step 1) Set up the structure in Fig. 2 with integer values $a_{k, \nu}$ and delays $\rho_{k, \nu}$ satisfying (46). The choice of $a_{k, \nu}$ and $\rho_{k, \nu}$ is arbitrary within the framework set by (46).

Step 2) Choose the lengths of filters $B_{k, \nu}(z)$ such that the desired final prototype filter length can be met.

Step 3) Using numerical optimization, find the coefficients of $B_{k, \nu}(z)$ such that the prototype's stopband attenuation or the coding gain of the resulting modulated filter bank is maximized.

Step 4) Round the coefficients of $B_{k, \nu}(z)$ to the nearest integers. From the lifting structure with integer factors $a_{k, \nu}$ and systems $B_{k, \nu}(z)$, obtain the polyphase components of the final integer prototype.

The size of the filter coefficients obtained with the above procedure is not explicitly controlled. It is mainly governed by the size of factors $a_{k, \nu}$. Generally, to obtain filters with small integer coefficients, the numbers $a_{k, \nu}$, should be small.

Remark: When implementing the polyphase filters, one can either directly use the structure given in Fig. 2(b) or implement them as transversal filters with the coefficients obtained from Step 4).

\section{Design of InTEGER Modulation SchemeS}

In this section, we present different methods for designing integer modulation schemes. First, we look at solutions that resemble the symmetry properties of cosine modulation, and then, we outline a novel projection-based method. The focus is on finding matrices that contain very small integers, thus allowing for low-cost implementations and applications like lossless compression. Other techniques such as the use of micro rotations [14], [24] or Householder matrices [10], [15] structurally guarantee PR, but they usually lead to integer matrices with extremely large entries.

\section{A. Integer Modulation That Resembles Cosine Modulation}

From the perfect reconstruction point of view, it is sufficient to find an integer matrix $\boldsymbol{V}$ satisfying (27) with a matrix $\boldsymbol{\Gamma}$, depending on the given case. However, for the performance of a filterbank, it is of crucial importance that the modulation sequences properly shift the prototype filters' frequency responses to the appropriate center frequencies, as demonstrated in Fig. 1(c), without corrupting the frequency selectivity of the filters. In the following, we accomplish this by creating integer sequences that have the same periodicities and symmetries as the original cosine sequences.

We consider the equation $c_{1, k}(m)=c_{1,0}(n)$ with $c_{1, k}(m)$ and $D$ according to (2) and (3), respectively, and solve it for $n$. This yields

$$
n=m(2 k+1)-k(2 s M+2 M-1)-M / 2+(-1)^{k} M / 2 .
$$

It is easy to see that $n$ is an integer if $m$ is an integer, which shows that the sequences $c_{1, k}(m)$ for $k=1,2, \ldots, M-1$ are permuted versions of $c_{1,0}(n)$. As a consequence, all elements $\left[\boldsymbol{V}^{c}\right]_{k, n}$ for $k>0$ can be derived from $\left[V^{c}\right]_{\mathrm{O}, n}$ through permutations, sign changes, and the possible introduction of additional zeros (only required for odd $M$ ). The structure of $\boldsymbol{V}^{c}$ is easily derived from (20) and (47) under consideration of the periodicity properties (14) and (15). Fig. 3 depicts the structure of $V^{c}$ for various $M$ and even $s$. The structures for odd $s$ are the left-right flipped versions of the ones for even $s$.

To construct a matrix $\boldsymbol{V}$ that resembles the original cosine matrix $V^{c}$, we choose the values $[V]_{0, n}, n=0, \ldots, M-1$ in such a way that they follow the shape of $\left[V^{c}\right]_{0, n}$ as closely as 


$$
\left[\begin{array}{rrr}
a & b & c \\
-a & 0 & a \\
-a & b & -c
\end{array}\right]\left[\begin{array}{rrrr}
a & b & c & d \\
-b & d & a & c \\
-c & a & -d & -b \\
d & -c & b & -a
\end{array}\right]\left[\begin{array}{rrrrr}
a & b & c & d & e \\
-a & -d & e & b & c \\
-a & 0 & a & 0 & -a \\
a & -d & -e & b & -c \\
a & -b & c & -d & e
\end{array}\right]\left[\begin{array}{rrrrrr}
a & b & c & d & e & f \\
-b & -e & e & b & b & e \\
-c & e & a & f & -b & -d \\
d & -b & -f & a & -e & -c \\
e & -b & b & -e & -e & b \\
-f & e & -d & c & -b & a
\end{array}\right]
$$

$$
\left[\begin{array}{rrrrrrr}
a & b & c & d & e & f & g \\
-a & -d & -g & f & c & b & e \\
-a & -f & e & b & g & -d & -c \\
a & 0 & -a & 0 & a & 0 & -a \\
a & -f & -e & b & -g & -d & c \\
-a & d & -g & -f & c & -b & e \\
-a & b & -c & d & -e & f & -g
\end{array}\right]\left[\begin{array}{rrrrrrrr}
a & b & c & d & e & f & g & h \\
-b & -e & -h & f & c & a & d & g \\
-c & -h & d & b & g & -e & -a & -f \\
d & -f & -b & h & a & g & -c & -e \\
e & -c & -g & a & -h & -b & f & d \\
-f & a & -e & -g & b & -d & -h & c \\
-g & d & -a & c & -f & -h & e & -b \\
h & -g & f & -e & d & -c & b & -a
\end{array}\right]
$$

Fig. 3. Structure of $\boldsymbol{V}^{c}$ for $M=3,4,5,6,7,8$ for even $s$. The structures for odd $s$ are the left-right flipped versions of the ones for even $s$.

possible and construct $V$ by using the given construction principle of $V^{c}$. To guarantee PR, the constructed matrix $V$ must satisfy (27) with $\boldsymbol{\Gamma}$ depending on the case under consideration. A search over integers in the range

$$
[V]_{0, n} \in\left\{\left\lfloor\alpha\left[V^{c}\right]_{0, n}\right\rfloor-\beta, \ldots,\left\lceil\alpha\left[V^{c}\right]_{0, n}\right\rceil+\beta\right\}
$$

with a suitable scalar $\alpha$ and a small integer $\beta$ is used to find the required values.

Compared with optimizing the matrix $V$ directly, which would result in a search over $M^{2}$ variables, by taking into consideration the structure of the matrix $\boldsymbol{V}^{c}$, we were able to reduce the search space to $M$ entries. In addition, the integer modulation matrix is more likely to follow the waveform of the cosine modulation.

\section{B. Projection-Based Matrix Design}

To design $\boldsymbol{V}$ satisfying (27), we start with a single arbitrary vector $\boldsymbol{v}_{1}$ that contains integer values and that will form one of the columns of $V^{T}$. The vector $\boldsymbol{v}_{1}$ may be an integer approximation of one of the columns of $\sqrt{\varepsilon / 2 M}\left[V^{c}\right]^{T}$. During the design procedure, we successively design integer vectors $\boldsymbol{v}_{k}, k=$ $2,3, \ldots M$, which are orthogonal to the sets $\boldsymbol{v}_{1}, \ldots, \boldsymbol{v}_{k-1}$. The procedure will first be described for even $M$, and then, the modifications for odd $M$ will be outlined.

Design procedure for even $M$ :

Step 1) Let $\varepsilon=\boldsymbol{v}_{1}^{T} \boldsymbol{v}_{1}$. Set $k=1$ and $A=\boldsymbol{v}_{1}$.

Step 2) Find a vector $\boldsymbol{v}_{k+1}$ that has the properties $\boldsymbol{A}^{T} \boldsymbol{v}_{k+1}=$ $\mathbf{0}, \boldsymbol{v}_{k+1}^{T} \boldsymbol{v}_{k+1}=\varepsilon$ and contains only integers. For this, one of the two following methods can be used.

Method 1: Set $\boldsymbol{v}_{k+1}=\boldsymbol{Z} \boldsymbol{v}_{k}$ with $\boldsymbol{Z}=$ $\boldsymbol{J}_{M} \operatorname{diag}[1,-1, \ldots, 1,-1]$. Note that this method can only be used during every second iteration.

Method 2: Compute the projection matrix

$$
B=\boldsymbol{I}_{M}-\boldsymbol{A}\left[A^{T} \boldsymbol{A}\right]^{-1} \boldsymbol{A}^{T}=\boldsymbol{I}_{M}-\frac{1}{\varepsilon} \boldsymbol{A} \boldsymbol{A}^{T} .
$$

Form a matrix $\boldsymbol{C}$ from a subset of columns of $\boldsymbol{B}$ and express the vector $\boldsymbol{v}_{k+1}$ as

$$
\boldsymbol{v}_{k+1}=\boldsymbol{C p}
$$

where $\boldsymbol{p}$ is a vector of integers that yields $\boldsymbol{v}_{k+1}^{T} \boldsymbol{v}_{k+1}=\varepsilon$, with $\boldsymbol{v}_{k+1}$ containing integers. The vector $\boldsymbol{v}_{k+1}$ is automatically orthogonal to $\boldsymbol{A}$. By gathering only a linearly independent subset of the columns of $\boldsymbol{B}$ in $\boldsymbol{C}$, the length of $\boldsymbol{p}$ and, thus, the number of unknowns can be reduced. The condition $\boldsymbol{v}_{k+1}^{T} \boldsymbol{v}_{k+1}=\varepsilon$ is usually met for a number of integer parameter vectors $\boldsymbol{p}$ so that a solution can be chosen that leads to a close match between $\boldsymbol{v}_{k+1}$ and one of the columns of $\sqrt{\varepsilon / 2 M}\left[V^{c}\right]^{T}$. To reduce the search effort, initial guesses $\tilde{\boldsymbol{p}}$ can be computed as $\tilde{\boldsymbol{p}}=\operatorname{round}\left(\alpha\left[\boldsymbol{C}^{T} \boldsymbol{C}\right]^{-1} \boldsymbol{C}^{T} \boldsymbol{v}_{k+1}^{c}\right)$. Here, $\boldsymbol{v}_{k+1}^{c}$ is a column of $\left[V^{c}\right]^{T}$ that is to be approximated, and $\alpha$ is a scale factor that ensures that $\tilde{\boldsymbol{p}}^{T} \boldsymbol{C}^{T} \boldsymbol{C} \tilde{\boldsymbol{p}} \approx \varepsilon$.

Step 3) Set $\boldsymbol{A}:=\left[\boldsymbol{A}, \boldsymbol{v}_{k+1}\right]$, and let $k:=k+1$. If $k=$ $M-1$, the procedure is finished, and $V=A^{T}$. Otherwise, go to Step 2).

Modifications for Odd $M$ : In Step 2), if $M$ is odd, then Method 1 cannot be applied, and Method 2 must be used throughout the algorithm. In addition, because of the requirements in (32) and (33), one needs to find $M-1$ vectors with energy $\varepsilon$ and one with energy $2 \varepsilon$. This can be achieved by first designing the $M-1$ vectors with equal energy and then changing the requirement to $\boldsymbol{v}_{M}^{T} \boldsymbol{v}_{M}=2 \varepsilon$ for the last vector. Alternatively, the algorithm can be used to first design a matrix $\boldsymbol{U}$ satisfying $\boldsymbol{U}^{T} \boldsymbol{U}=(\varepsilon / 2) \boldsymbol{I}$. $\boldsymbol{U}$ can then be used to construct $T_{1}$ according to (34), and $V$ can be found as $V=T_{1} Y_{1}^{+}$, as outlined in the remarks in Section III-A.

Combined Method: When aiming to design a matrix $V$ with the method in Section V-A and only $M_{0}<M$ rows can be found that follow the symmetry of the cosine sequences and are orthogonal, then the method in this section may be used to obtain the remaining $M-M_{0}$ orthogonal vectors (rows) that are required to complete the matrix $V$. 
TABLE I

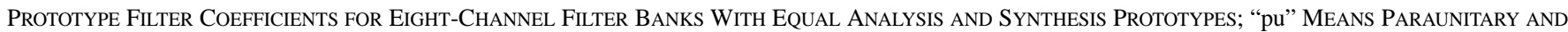
"bi” STANDS FOR BIORTHOGONAL

\begin{tabular}{c|c|l}
\hline Filter No. & Parameters & Filter Coefficients $p(n)=q(n)$ \\
\hline 1 & $M=8, \mathrm{pu}, L=32, D=31$ & $\{-6,-4,0,-6,7,0,8,17,24,33,41,48,56,62,66,68$, \\
& & $68,66,62,56,48,41,33,24,17,8,0,7,-6,0,-4,-6\}$ \\
\hline 2 & $M=8, \mathrm{bi}, L=32, D=15$ & $\{32,40,48,56,71,76,74,76,96,88,80,72,55,44$, \\
& & $42,28,8,8,0,0,0,0,2,3,-8,-8,0,0,0,0,2,3\}$ \\
\hline 3 & $M=8, \mathrm{pu}, L=16, D=15$ & $\{1,1,1,1,2,2,2,2,2,2,2,2,1,1,1,1\}$ \\
\hline
\end{tabular}

\section{DESIGN EXAMPLES}

This section presents design examples for paraunitary and biorthogonal filterbanks with even and odd numbers of channels and compares filterbanks in an image coding example. The coefficient size of prototypes and modulation sequences are small to moderate to obtain a low implementation cost. Typically, filterbanks with larger coefficients result in better performance, and the aim is to design the prototype and modulation with best performance for the given bit length. The bit length should also be taken into account when comparing the performance of different filterbanks in a given application.

\section{A. Example 1-Paraunitary Filter Bank $M=8, L=32$}

The first example considers an eight-channel, paraunitary filter bank with a prototype filter of length $L=32$. Since the prototype filter has to be linear phase, the delay is $D=31$, which corresponds to $s=1$. The integer coefficients of the prototype have been found using [8] and are listed in Table I as Filter 1.

Using the method in Section $\mathrm{V}$-A, we find the following integer values for the first row of $V$ that results in PR:

$$
\{5,9,14,19,23,24,28,27\} .
$$

The matrix $V$ is constructed according to the symmetries given in Fig. 3 for $M=8$ and $s$ odd and becomes

$$
\begin{aligned}
& V= \\
& {\left[\begin{array}{rrrrrrrr}
5 & 9 & 14 & 19 & 23 & 24 & 28 & 27 \\
9 & 23 & 27 & 24 & 14 & -5 & -19 & -28 \\
-14 & -27 & -19 & 9 & 28 & 23 & -5 & -24 \\
-19 & -24 & 9 & 27 & 5 & -28 & -14 & 23 \\
23 & 14 & -28 & -5 & 27 & -9 & -24 & 19 \\
24 & -5 & -23 & 28 & -9 & -19 & 27 & -14 \\
-28 & 19 & -5 & -14 & 24 & -27 & 23 & -9 \\
-27 & 28 & -24 & 23 & -19 & 14 & -9 & 5
\end{array}\right] .}
\end{aligned}
$$

To allow a comparison with the original cosine matrix $V^{c}$, the first row of $V^{c}$ has been scaled by a factor $\alpha$ such that $\sum_{n=0}^{7}$ $\alpha\left[\boldsymbol{V}^{c}\right]_{0, n}-[\boldsymbol{V}]_{0, n}=0$ and rounded to two digits, resulting in $\{2.86,8.48,13.77,18.53,22.58,25.76,27.95,29.07\}$. The maximum difference between $\left[V^{c}\right]_{0, n}$ and $[V]_{0, n}$ then turns out to be 2.14 .

The frequency responses of the modulated filters are depicted in Fig. 4, together with the ones for the ELT filters according to [25]. To enable easier comparisons, the gains of the filters have been adjusted so that the average maximum gain of all filters in a graph is $0 \mathrm{~dB}$. As the plots show, the frequency selectivity and stopband attenuation of the integer filters are in the same range as for the ELT.

In addition to the stopband attenuation, the coding gain is an important indication of the quality of a filterbank [26], [27]. For an $\operatorname{AR}(1)$ process with correlation coefficient $\rho=0.95$, the coding gain of the above filterbank is $9.06 \mathrm{~dB}$. This is slightly more than the gain obtained by a real-valued eight-point DCT, which yields $8.83 \mathrm{~dB}$ and almost as much as that of the ELT, which provides a gain of $9.39 \mathrm{~dB}$ [25].

\section{B. Example 2-Biorthogonal Low-Delay Filterbank $M=8$, $L=32$}

To reduce the delay of the filterbank in Example 1 to $D=15$ while keeping the filter length constant, a biorthogonal prototype has been designed with the method in Section IV. The objective function used during optimization was the coding gain. The coefficients are listed in Table I, Filter 2.

We use the flipped version of (50) as the first row of $V$, and this time, we have to consider the symmetries for even $s$ in Fig. 3. The frequency responses of the modulated filters are depicted in Fig. 5. The comparison between Figs. 4 and 5 shows that the reduction in delay comes at the cost of a slightly decreased stopband attenuation. The coding gain for the same $\mathrm{AR}(1)$ process as in Example 1 is $8.91 \mathrm{~dB}$. Thus, the coding gain is slightly reduced, compared with Example 1.

\section{Example With Very Small Filter Coefficients}

In this example, we consider a paraunitary filter bank where both the modulation matrix and the prototype filter coefficients have very small integer numbers, thus resulting in implementations with small word lengths. This filterbank can be applied to lossless coding since it only slightly increases the wordlength of the subband signals compared with the input signal. The prototype is listed as number 3 in Table I. Using the method in Section $\mathrm{V}-\mathrm{B}, V$ has been found as

$$
\boldsymbol{V}=\left[\begin{array}{rrrrrrrr}
1 & 1 & 1 & 1 & 3 & 3 & 3 & 3 \\
-3 & -3 & -3 & -3 & 1 & 1 & 1 & 1 \\
1 & 1 & -1 & -1 & -3 & -3 & 3 & 3 \\
3 & 3 & -3 & -3 & 1 & 1 & -1 & -1 \\
1 & -1 & -1 & 1 & 3 & -3 & -3 & 3 \\
-3 & 3 & 3 & -3 & 1 & -1 & -1 & 1 \\
-1 & 1 & -1 & 1 & 3 & -3 & 3 & -3 \\
-3 & 3 & -3 & 3 & -1 & 1 & -1 & 1
\end{array}\right]
$$

The frequency responses of the modulated filters are depicted in Fig. 6. Note that seven of the modulated filters exactly have 


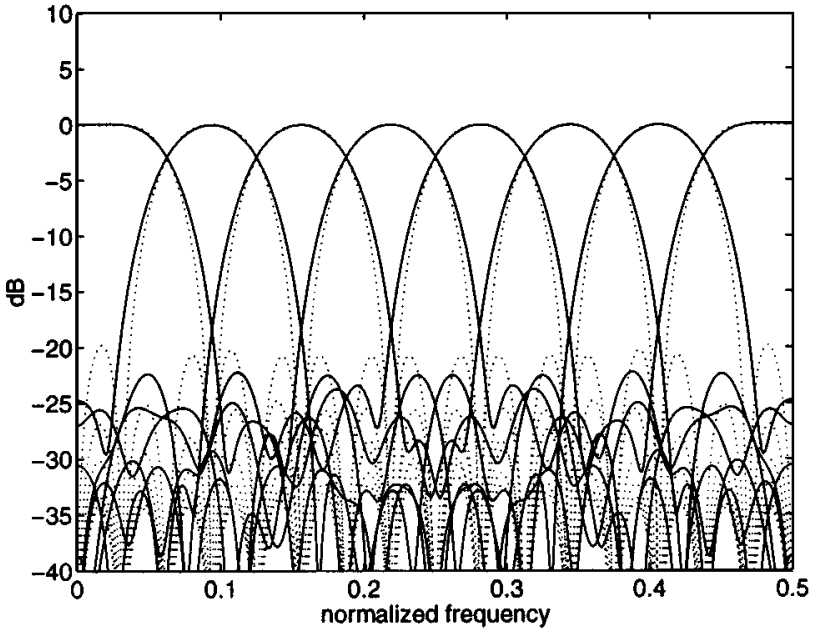

Fig. 4. Normalized frequency responses of integer-modulated, paraunitary eight-channel filterbanks with filter length $L=32$ and delay $D=31$. Prototype number 1 of Table I and $V$ constructed from the sequence in (50). The dotted lines show the frequency responses of the ELT filters of [25].

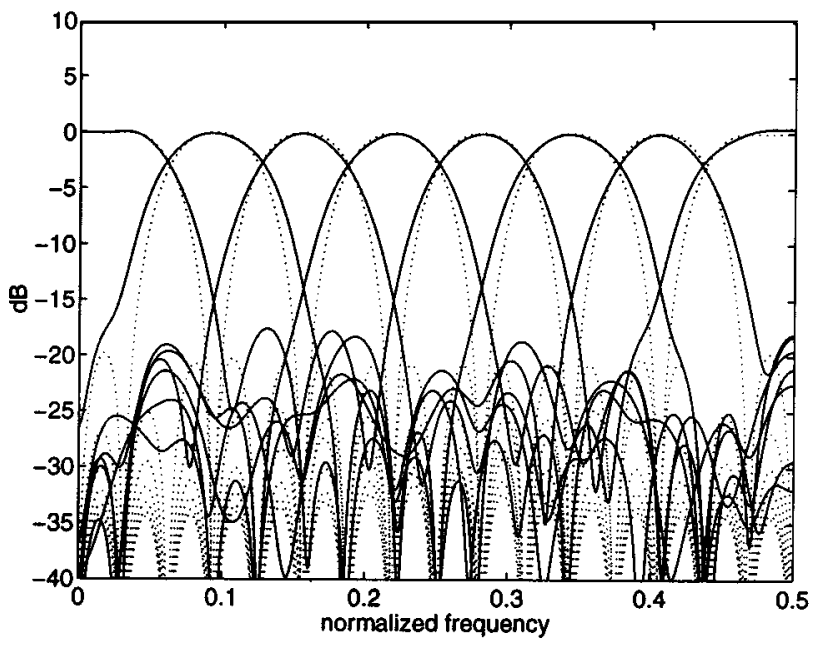

Fig. 5. Normalized frequency responses of integer-modulated, biorthogonal eight-channel filterbanks with filter length $L=32$ and delay $D=15$. Prototype number 2 of Table I and $V$ constructed from (50) and Fig. 3 with even $s$. The dotted lines show the frequency responses of the ELT.

zero mean, which means that the dc behavior is perfect. The maximum coefficient of the final modulated filters is 6 , and the coding gain is $8.1 \mathrm{~dB}$. The stopband attenuation of most filters is around $6 \mathrm{~dB}$, which is quite high for such small filter coefficients.

\section{Example of Filterbank With an Odd Number of Channels}

This example demonstrates the design of biorthogonal filterbanks with an odd number of channels. The chosen number of channels is $M=5$, the overall system delay is $D=9$, and the prototype filter length is $L=20$. With the method described in Section IV, the following low-delay prototype satisfying (30) has been found:

$p(n)=\{10,20,30,40,50,52,53,49,41,34,20,12,0$

$$
-4,-5,-8,-6,0,-2,-2\} \text {. }
$$

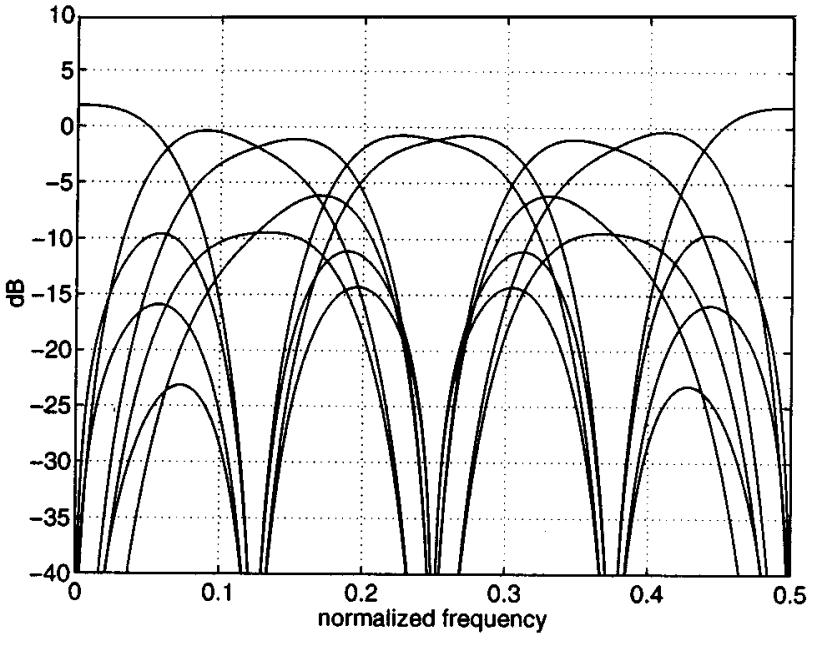

Fig. 6. Normalized frequency responses. Paraunitary filterbank $M=8, L=$ $16, D=15$, prototype number 3 in Table I with $\boldsymbol{V}$, as in (52).

A suitable matrix $V$ has been found using a combination of the methods in Sections V-A and V-B, as outlined in the remark in Section V-B. The matrix is

$$
V=\left[\begin{array}{rrccc}
30 & 30 & 25 & 16 & 9 \\
-30 & -16 & 9 & 30 & 25 \\
-32 & 0 & 30 & 0 & -30 \\
30 & -16 & -9 & 30 & -25 \\
30 & -30 & 25 & -16 & 9
\end{array}\right]
$$

Note that all rows of $\boldsymbol{V}$ in (53), except the third one, resemble the symmetries found in the corresponding cosine matrix $\boldsymbol{V}^{c}$. The frequency responses of the resulting analysis filters for $M=5$ are depicted in Fig. 7.

\section{E. Example of Coupled Filterbank Design}

We consider a four-channel filter bank with symmetric, paraunitary prototype of length $L=8$. Using the method in Section $\mathrm{V}$-A, a modulation matrix $V$ has been found as

$$
\boldsymbol{V}=\left[\begin{array}{rcrr}
10 & 10 & 5 & 2 \\
-11 & 6 & 8 & 3 \\
-2 & 5 & -10 & 10 \\
-3 & 8 & -6 & -11
\end{array}\right]
$$

It satisfies $V^{T} V=\operatorname{diag}[234,225,225,234]$, which means that $w_{0}=225, w_{1}=234$. A suitable prototype is given by

$$
p(n)=\{1,3,4,5,5,4,3,1\} .
$$

It is easy to see that (39) is satisfied with $\lambda_{k}=1, k=0,1$, resulting in

$$
\gamma=w_{0}\left(p^{2}(0)+p^{2}(3)\right)=w_{1}\left(p^{2}(1)+p^{2}(2)\right)=5850 .
$$

Because of equal values for $\lambda_{k}, k=0,1$, the same prototype is to be used for analysis and synthesis. The frequency responses of the resulting filters are depicted in Fig. 8. One can see that the filters have very good frequency selectivity. Interestingly, the entire filterbank is paraunitary, although the matrix $V$ is not orthogonal, and the prototype cannot be used for a paraunitary cosine-modulated filterbank. 


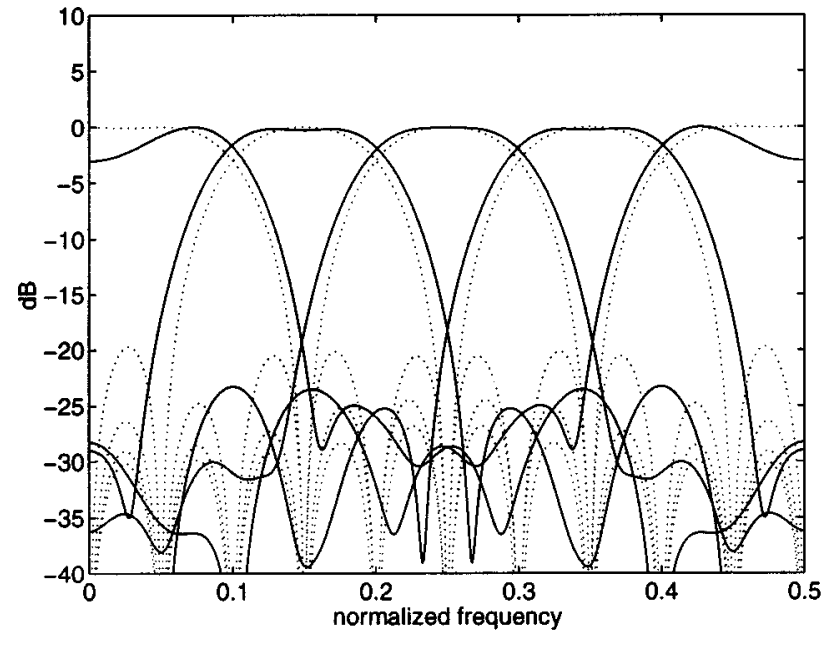

Fig. 7. Normalized frequency responses of integer-modulated, five-channel filter bank with filter length $L=20$ and delay $D=9$. The dotted lines show the frequency responses of the ELT, which has filter length $L=20$ and delay $D=19$.

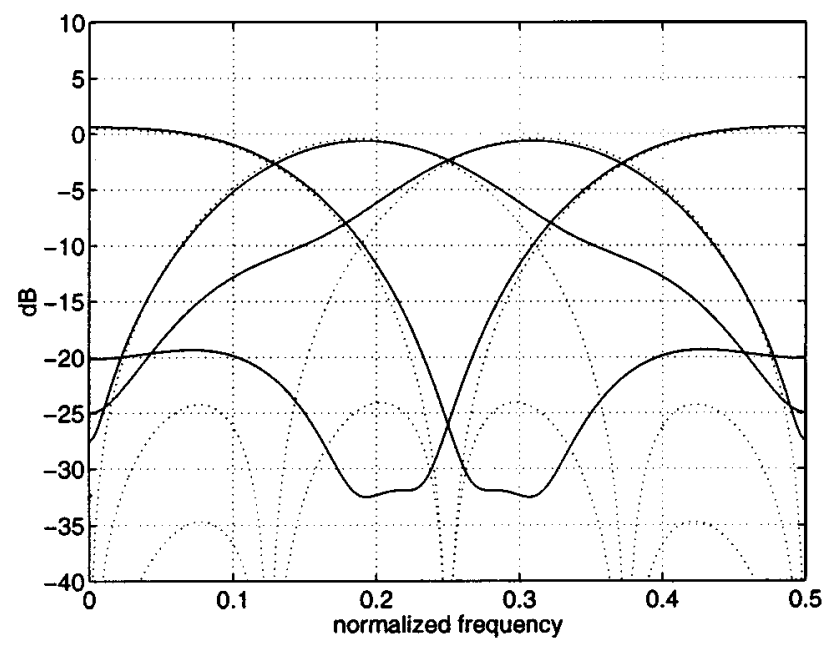

Fig. 8. Normalized frequency responses of integer-modulated, four-channel filter bank with coupled design. The dotted lines show the frequency responses of the MLT filters of [22].

\section{F. Image Compression Results}

To demonstrate an application of the proposed integer-modulated filterbanks, we consider the problem of image compression. The test configuration is as follows. A test image has been decomposed with 2-D, 64-channel, separable filterbanks derived from 1-D, eight-channel, modulated filterbanks. For lossy compression of the transform coefficients an algorithm known as set partitioning in hierarchical trees (SPIHT) [28] with the virtual extension of [29] has been used. The SPIHT algorithm is one of the best compression algorithms for wavelet-based coders, but as demonstrated in [30], it can equally well be used to compress the coefficients of uniform transforms such as cosine-modulated filterbanks.

Tests have been carried out for the filterbanks presented in Sections VI-A and VI-C and for the eight-channel MLT and ELT [22]. Table II shows the coding results for the test image "Goldhill" of size $512 \times 512$. The distortion of the lossy coder is measured in form of the peak signal-to-noise ratio (PSNR).
TABLE II

PSNRS FOR CODING THE GOLDHILl IMAGE WITH DIFFERENT FILTER BANKS AT VARIOUS BIT RATES

\begin{tabular}{c|c|c|c|c}
\hline & \multicolumn{4}{|c}{ Bit Rate [bits per pixel] } \\
\hline Filter bank & 0.125 & 0.25 & 0.5 & 1 \\
\hline MLT & 26.51 & 29.13 & 32.07 & 35.52 \\
ELT & 26.16 & 28.91 & 31.84 & 35.28 \\
Section VI-A & 25.96 & 28.35 & 31.15 & 34.77 \\
Section VI-C & 25.72 & 28.01 & 30.62 & 33.92 \\
\hline
\end{tabular}

As could be expected, the best performance is obtained with the MLT and ELT using real arithmetic, followed by the filterbank of Section VI-A with moderately sized integers. However, even the filterbank of Section VI-C, which has extremely small coefficients, performs remarkably well.

\section{COMPUTATIONAL COMPLEXITY}

When comparing the proposed integer-modulated filterbanks with cosine-modulated filterbanks in terms of computational complexity, their main advantage is the use of fast integer multiplications instead of more complex floating-point multiplications. In addition, since the coefficients are generally small and, thus, have a small number of ones in their binary representation, the integer multiplications can be replaced by shift and add operations.

However, the integer-modulated filterbanks with the modulation matrices designed according to Section $\mathrm{V}$ do not have a fast realization for the transform, resulting in a computational cost of order $\mathcal{O}\left(M^{2}\right)$ compared with $\mathcal{O}(M \log M)$ for the fast DCT of a cosine-modulated filterbank. They are therefore best suited for filterbanks with a small to moderate number of subbands.

Integer modulation matrices with computational cost of the order $\mathcal{O}(M \log M)$ can be designed as in [14] and [24]. Their drawback is that they typically contain extremely large integers. On the other hand, these approaches can be used to design modulation matrices for very large $M$ when the construction principles described in Section V become computationally too expensive.

The prototype design method described in Section IV can easily be used for any $M$ so that this prototype design, in conjunction with a modulation design as in [14] and [24], allows the design of integer filterbanks for a large number of bands that have both the advantage of integer arithmetic and a fast modulation with a cost of order $\mathcal{O}(M \log M)$.

\section{CONCLUSIONS}

We have derived the PR conditions for general (noncosine) modulated filterbanks including paraunitary and low-delay, biorthogonal filterbanks. The presented conditions hold for even and odd numbers of channels and include designs where the conditions on the prototype and the modulation are either decoupled or coupled. A lifting scheme has been introduced for the design of biorthogonal prototypes. New algebraic methods have been presented for the construction of suitable modulation sequences. Design examples were presented that show filterbanks with excellent properties and extremely small integer coefficients. 


\section{REFERENCES}

[1] M. Antonini, M. Barlaud, P. Mathieu, and I. Daubechies, "Image coding using wavelet transform," IEEE Trans. Image Processing, vol. 1, pp. 205-220, Apr. 1992.

[2] B. R. Horng, H. Samueli, and A. N. Wilson, Jr., "The design of two-channel lattice-structure perfect-reconstruction filterbanks using powers-of-two coefficients," IEEE Trans. Circuits Syst., vol. 40, pp. 497-499, July 1993.

[3] A. N. Akansu, "Multiplierless PR quadrature mirror filters for subband image coding," IEEE Trans. Image Processing, vol. 9, pp. 1359-1363, Sept. 1996.

[4] S. Sriranganathan, D. R. Bull, and D. W. Redmill, "The design of low complexity two-channel lattice-structure perfect-reconstruction filterbanks using genetic algorithms," in Proc. IEEE Int. Symp. Circuits Syst., vol. 4, Hong Kong, June 1997, pp. 2393-2396.

[5] W. Sweldens, "The lifting scheme: A custom design construction of biorthogonal wavelets," J. Appl. Comput. Harmonic Anal., vol. 3, no. 2, pp. 186-200, 1996.

[6] R. Calderbank, I. Daubechies, W. Sweldens, and B.-L. Yeo, "Wavelet transforms that map integers to integers," Appl. Comput. Harmon. Anal., vol. 5, no. 3, pp. 332-369, 1998

[7] T. Karp, A. Mertins, and T. Q. Nguyen, "Efficiently VLSI-realizable prototype filters for modulated filterbanks," in Proc. IEEE Int. Conf. Acoust., Speech, Signal Process., vol. 3, Munich, Germany, Apr. 1997, pp. $2445-2448$

[8] A. Mertins, "Subspace approach for the design of cosine-modulated filterbanks with linear-phase prototype filter," IEEE Trans. Signal Processing, vol. 46, pp. 2812-2818, Oct. 1998.

[9] M. Bi, S. H. Ong, and Y. H. Ang, "Integer-modulated FIR filterbanks for image compression," IEEE Trans. Circuits Syst. Video Technol., vol. 8, pp. 923-927, Dec. 1998

[10] A. Mertins, T. Karp, and J. Kliewer, "Design of perfect reconstruction integer-modulated filterbanks," in Proc. Int. Symp. Signal Process. Appl., vol. 2, Brisbane, Australia, Aug. 1999, pp. 591-594.

[11] _ , "Integer-modulated filterbanks providing perfect reconstruction," in Proc. Eur. Signal Process. Conf., Tampere, Finland, Sept. 2000, pp. $1763-1766$.

[12] S. C. Chan, W. Liu, and K. L. Ho, "Perfect reconstruction modulated filterbanks with sum-of-powers-of-two coefficients," in Proc. IEEE Int. Symp. Circuits Syst., vol. 2, Geneva, Switzerland, May 2000, pp. 73-76.

[13] W. K. Cham, "Development of integer cosine transforms by the principle of dyadic symmetry," Proc. Inst. Elect. Eng., pt. I, vol. 136, pp. 276-282, Aug. 1989.

[14] J. Liang and T. D. Tran, "Fast multiplierless approximation of the DCT with the lifting scheme," in Proc. SPIE Appl. Digital Image Process., San Diego, CA, Aug. 2000.

[15] P. P. Vaidyanathan, Multirate Systems and filterbanks. Englewood Cliffs, NJ: Prentice-Hall, 1993.

[16] M. Vetterli and J. Kovačević, Wavelets and Subband Coding. Englewood Cliffs, NJ: Prentice-Hall, 1995.

[17] G. Strang and T. Nguyen, Wavelets and filterbanks. Wellesley, MA: Wellesley-Cambridge, 1996.

[18] G. D. T. Schuller and M. J. T. Smith, "A new framework for modulated perfect reconstruction filterbanks," IEEE Trans. Signal Processing, vol. 44, pp. 1941-1954, Aug. 1996

[19] J. Kliewer and A. Mertins, "Oversampled cosine-modulated filterbanks with arbitrary system delay," IEEE Trans. Signal Processing, vol. 46, pp. 941-955, Apr. 1998.

[20] H. Bölcskei and F. Hlawatsch, "Oversampled cosine modulated filterbanks with perfect reconstruction," IEEE Trans. Circuits Syst. II, vol. 45, pp. 1057-1071, Aug. 1998.

[21] P. N. Heller, T. Karp, and T. Q. Nguyen, "A general formulation for modulated filterbanks," IEEE Trans. Signal Processing, vol. 47, pp. 986-1002, Apr. 1999.
[22] H. S. Malvar, Signal Processing With Lapped Transforms. Norwood, MA: Artech House, 1992

[23] S. C. Chan and C. W. Kok, "Perfect reconstruction modulated filterbanks without cosine constraints," in Proc. IEEE Int. Conf. Acoust., Speech, Signal Process., vol. 3, Minneapolis, MN, Apr. 1993, pp. 189-192.

[24] P. Rieder, J. Götze, M. Sauer, and J. A. Nossek, "Orthogonal approximation of the discrete cosine transform," in Proc. Eur. Conf. Circuit Theory Des., Istanbul, Turkey, Aug. 1995, pp. 1003-1006.

[25] H. S. Malvar, "Extended lapped transforms: Fast algorithms and applications," IEEE Trans. Signal Processing, vol. 40, pp. 2703-2714, Nov. 1992.

[26] N. S. Jayant and P. Noll, Digital Coding of Waveforms. Englewood Cliffs, NJ: Prentice-Hall, 1984.

[27] J. Katto and Y. Yasuda, "Performance evaluation of subband coding and optimization of its filter coefficients," Proc. SPIE Visual Commun. Image Process., pp. 95-106, Nov. 1991.

[28] A. Said and W. A. Pearlman, "A new fast and efficient image codec based on set partitioning in hierarchical trees," IEEE Trans. Circuits Syst. Video Technol., vol. 6, pp. 243-250, June 1996.

[29] E. Khan and M. Ghanbari, "Very low bit rate video coding using virtual SPIHT," Electron. Lett., vol. 37, pp. 40-42, Jan. 2001.

[30] T. D. Tran and T. Q. Nguyen, "A progressive transmission image coder using linear phase uniform filterbanks as block transforms," IEEE Trans. Image Processing, vol. 8, pp. 1493-1507, Nov. 1999.

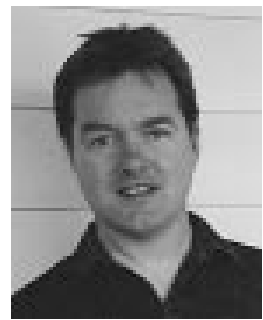

Alfred Mertins (M'94) received the Dipl.-Ing. degree from the University of Paderborn, Paderborn, Germany, in 1984 and the Dr.-Ing. degree in electrical engineering and the Dr.-Ing. habil. degree in telecommunications from the Hamburg University of Technology, Hamburg, Germany, in 1991 and 1994, respectively.

From 1986 to 1991, he was with the Hamburg University of Technology, from 1991 to 1995 with the Microelectronics Applications Center Hamburg, from 1996 to 1997 with the University of Kiel, Kiel, Germany, and from 1997 to 1998 with the University of Western Australia, Perth. Since 1998, he has been with the School of Electrical, Computer, and Telecommunications Engineering, University of Wollongong, Wollongong, Australia. His research interests include digital signal processing, wavelets and filterbanks, image and video processing, and digital communications.

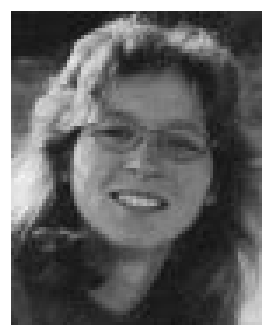

Tanja Karp (M'98) received the Dipl.-Ing. and the Dr.-Ing. degrees in electrical engineering from Hamburg University of Technology, Hamburg, Germany, in 1993 and 1997, respectively.

In 1995 and 1996, she spent two months as a Visiting Researcher at the Signal Processing Department, ENST, Paris, France, and at the Mutirate Signal Processing Group, University of Wisconsin, Madison, respectively, working on modulated filterbanks. In 1997, she joined Mannheim University, Mannheim, Germany, as a Senior Research and Teaching Associate. From 1998 to 1999, she taught as a Guest Lecturer at Freiburg University, Freiburg, Germany. Since 2000, she has been an Assistant Professor with the Department of Electrical and Computer Engineering, Texas Tech University, Lubbock. Her research interests include multirate signal processing, filterbanks, audio coding, multicarrier modulation, and signal processing for communications.

Dr. Karp regularly reviews articles for several IEEE and EURASIP transactions. 